\title{
Clinical development of neridronate: potential for new applications
}

This article was published in the following Dove Press journal:

Therapeutics and Clinical Risk Management

3 April 2013

Number of times this article has been viewed

\section{Davide Gatti \\ Maurizio Rossini \\ Ombretta Viapiana \\ Luca Idolazzi \\ Silvano Adami}

Rheumatology Unit, Department of Medicine, University of Verona, Verona, Italy
Correspondence: Davide Gatti Rheumatology Unit, Department of Medicine, University of Verona, Piazzale Stefani I, 37126 Verona, Italy Tel +390456338607

Fax +390458123155

Email davide.gatti@univr.it
Abstract: Neridronate is an aminobisphosphonate, licensed in Italy for the treatment of osteogenesis imperfecta (OI) and Paget's disease of bone (PDB). A characteristic property of neridronate is that it can be administered both intravenously and intramuscularly, providing a useful system for administration in homecare. In this review, we discuss the latest clinical results of neridronate administration in OI and PDB, as well as in osteoporosis and other conditions. We will focus in particular on the latest evidence of the effect of neridronate on treatment of complex regional pain syndrome type I.

Keywords: bisphosphonates, osteogenesis imperfecta, osteoporosis, complex regional pain syndrome

\section{Background}

Findings of a recent study are shedding new light on neridronate (6-amino-1-hydroxyhexylidene-1,1-bisphosphonate), an aminobisphosphonate widely used in the treatment of bone diseases and with specific therapeutic indications for osteogenesis imperfecta (OI) and Paget's disease of bone (PDB) in Italy. Differently from other bisphosphonates, neridronate can be administered both intravenously and intramuscularly. The latter regimen has demonstrated to be particularly relevant in particular patient groups as its administration does not require hospitalization and is suitable for homecare.

Results from a recent multicenter interventional trial have demonstrated its efficacy also in the treatment of the complex regional syndrome type I, thus opening the way to new therapeutic indications.

Here we review the potential clinical uses of neridronate in several bone-related diseases, in light of most recent literature. A systematic review of clinical studies and randomized controlled trials on neridronate in bone diseases was carried out. This study is upgrading our previous work by reviewing papers published after 2009 . $^{1}$

\section{Chemical features}

A characteristic of all bisphosphonates is their P-C-P chain, ${ }^{2}$ where the negative charge of the phosphate groups attributes the compounds a high affinity for the positively charged calcium ions on the surface of the bone. The other two side chains that the carbon atom bears determine the biological activity of the bisphosphonate ${ }^{2}$ and its categorization in the two classes of nitrogen-containing (pamidronate, alendronate, ibandronate, neridronate, and olpadronate) or non-nitrogen containing (clodronate, etidronate, and tiludronate) drugs. 
The structure and three-dimensional conformation of these side chains determine the biological activity of the bisphosphonate, ${ }^{3}$ with the aminobisphosphonates having a higher potency than the non-aminobisphosphonates.

The aminobisphosphonates inhibit farnesyl pyrophosphate synthase, a key enzyme in the mevalonate pathway, which is required for the prenylation of proteins that play key roles in intracellular signaling pathways that regulate processes fundamental to osteoclast function. ${ }^{4}$

Belonging to the aminobisphosphonates, neridronate distinguishes itself for its intramuscular or intravenous formulations, which altogether prevent a number of complications entailed by oral bisphosphonates. In fact, although oral bisphosphonates are the treatment of choice for a variety of bone diseases, their administration bears an uncomfortable burden for patients, with inevitable consequences on compliance to therapy. Notoriously oral bisphosphonates can cause local irritation and ulceration of the esophagus and stomach, ${ }^{5}$ and for this reason they require a large amount of water when ingested and must be taken in an upright posture.

Pharmacokinetic studies have indicated that bisphosphonates have low oral absorption. The oral bioavailability of bisphosphonates is lower than $1 \%^{6}$ and therefore must be taken when fasting and separated from intake of food or other drugs. These difficulties in oral administration have indeed favored the development of parenteral formulations of bisphosphonates.

\section{Clinical use of neridronate}

Over the years, neridronate has proven efficacious for offlabel treatment of many clinical bone-related pathologies, other than OI and PDB. ${ }^{1}$ The following sections are an overview of the bone-related pathologies in which neridronate has been used to date and the most relevant underlying studies.

\section{Osteogenesis imperfecta}

OI is a hereditary disease caused by a collagen defect, mostly caused by mutations of the genes coding the chains of collagen type 1 (gene COL1A1 or COL1A2), which lead usually to autosomal dominant OI. The disorder causes increased bone fragility and low bone mass and deformity, and the severity of the disease varies extensively among patients.

Treatment with bisphosphonates, in particular oral treatment with pamidronate, was first reported on a 12-year-old child in 1987 by Devogelaer et al. ${ }^{7}$ Since then, bisphosphonates (generally pamidronate) administered intravenously have become the common treatment in children, ${ }^{8}$ with clinical evidence of significant increases in bone mineral density (BMD) and decrease in fracture incidence. ${ }^{9-11}$

Neridronate has been extensively investigated in patients with OI. In growing children, the neridronate treatment induces a rapid increase in BMD and a significant $64 \%$ decrease in fracture numbers, ${ }^{12}$ and similar results have been obtained also in newborns ( $<12$ months old) affected by the more severe forms of the disease, with some evidence of improvement in the rate of skeletal growth. ${ }^{13}$ The treatment has also been tested in adults with OI, with evidence of efficacy in lowering fracture rate. ${ }^{14}$

The drug was administered intravenously every 3 months diluted in $250 \mathrm{~mL}$ of saline solution and infused intravenously over 30 minutes. ${ }^{12-14}$ The dose was $2 \mathrm{mg} / \mathrm{kg}$ bodyweight, until a maximum dosage of $100 \mathrm{mg}$ neridronate in adult patients.

Recently, Semler et al, ${ }^{15}$ in a study involving 28 children with severe or moderate OI, compared the treatment effect of the bisphosphonates pamidronate and neridronate. Both bisphosphonates are administered parenterally, the former given on 3 consecutive days every 4 months, the latter once every 3 months. To evaluate the effect of the bisphosphonates on the skeletal structure of OI patients, the authors focused on changes of the vertebral morphometry.

Pamidronate and neridronate proved equally efficient in improving vertebral area and vertebral indices of patients with OI (Figure 1). The huge benefit in treating patients with neridronate instead of pamidronate is the reduced time of

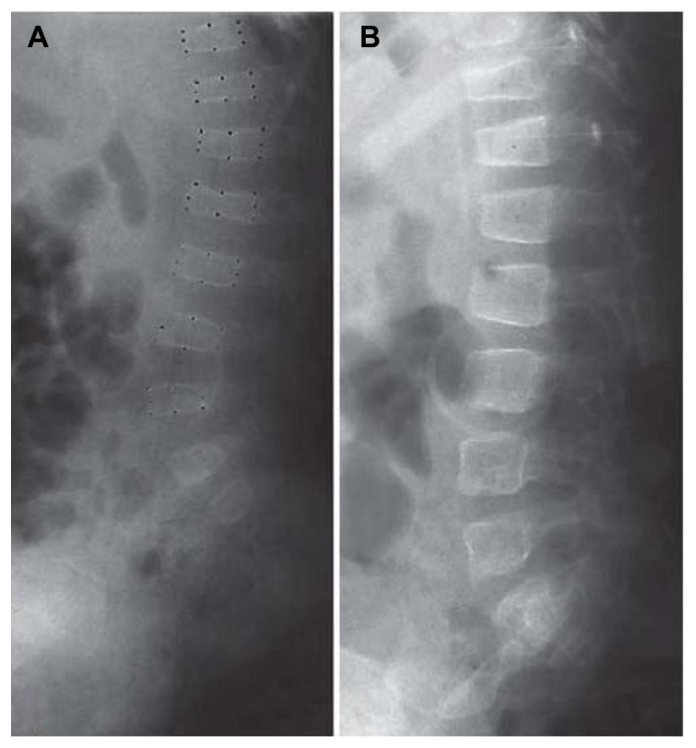

Figure I Vertebral bodies before (A) and after (B) treatment for 14 months for a child with Type I osteogenesis imperfecta.

Note: Copyright (C 20I I. Karger Publishers, Basel, Switzerland. Semler O, Beccard R, Palmisano D, et al. Reshaping of vertebrae during treatment with neridronate or pamidronate in children with osteogenesis imperfecta. Horm Res Paediatr. 201 1;76(5): $321-327 .{ }^{15}$ 
hospitalization. This is extremely important for the patients and their families and should lead to an increased quality of life in these severely handicapped children.

As an agent increasing bone apposition, or increasing statural growth, growth hormone $(\mathrm{GH})$ may play a therapeutic role in the treatment of OI. However, only few studies have been performed treating OI patients with GH. ${ }^{16-18}$

Recently, Antoniazzi et al have conducted a randomized controlled clinical trial evaluating the effects of a combined treatment of neridronate and recombinant $\mathrm{GH}(\mathrm{rGH})$ in children with mild and moderate OI. ${ }^{19}$ The goal of the study was to investigate whether the combination of neridronate and rGH can further improve bone metabolism of children with OI who are already receiving treatment with neridronate. In this study, 30 prepubertal children, already in therapy with neridronate, were randomized to continue treatment with neridronate alone or to initiate a 12-month treatment combining neridronate and $\mathrm{rGH}$. The effect of the combination of drugs was encouraging, as BMD at the lumbar spine and wrist as well as in the lumbar spine projected area increased significantly $(P<0.05)$. Moreover, the rate of linear growth velocity increased significantly with respect to the neridronate alone group.

A potential negative effect and serious complication of bisphosphonate therapy is osteonecrosis of the jaw, more commonly associated with intravenous administration of the drug. ${ }^{20}$ However, although unusual cases have been reported in adults using aminobisphosphonates, no case of osteonecrosis of the jaw has been reported in patients treated with neridronate. Maines et al studied the occurrence of osteonecrosis of the jaw in pediatric patients in treatment with neridronate for a period ranging from 1.0 to 12.9 years. No evidence of osteonecrosis of the jaw could be demonstrated, even in those patients who had been treated with neridronate for the longer period. ${ }^{21}$

\section{Paget's disease of bone}

PDB is the most common metabolic bone disease after osteoporosis. To date, the etiology of the disease is still unclear, and both environmental factors and genetic susceptibility are thought to be involved. ${ }^{22}$ The disease is characterized by focal increase of bone remodeling and disorganization of normal lamellar structure of the tissue, with consequent susceptibility to bone fractures, pain, and deformities.

PDB may involve one or more bones, and its distinctive feature is the large osteoclasts actively resorbing the bone. These cells seem to be adequately responding to bisphosphonate therapy, which therefore represents the treatment of choice. In particular, aminobisphosphonates are the most used PDB treatment. The response to treatment has been based on the changes in bone alkaline phosphatase (bAP) and variably defined by the full normalization of bAP or a decrease by $>75 \%$.

Neridronate is licensed in Italy for the treatment of Paget's disease at the dose of $100 \mathrm{mg}$ dissolved in $250-500 \mathrm{~mL}$ of saline solution given intravenously for 2 consecutive days. The registration was achieved after a multicenter clinical trial testing four different doses: 25, 50, 100, and $200 \mathrm{mg} .{ }^{23}$ The highest dose was the most effective and was associated with a $65 \%$ rate of full remission and a biochemical response (decrease of $>75 \%$ ) in $95 \%$ of the patients. ${ }^{23}$

Only few randomized clinical trials have compared the safety and efficacy of different bisphosphonates in PDB treatment. Namely, a comparison between oral alendronate and intravenous pamidronate proved that bisphosphonates had similar efficacy in achieving biochemical remission in previously untreated patients, but in those who had formerly been treated with pamidronate, alendronate was more effective. ${ }^{24}$ In two other studies, a single intravenous dose of zoledronic acid proved more effective than a 2-month course of oral administration of risedronate in controlling bone turnover in the short ${ }^{25}$ and long ${ }^{26}$ term.

In a study conducted in 2007, Merlotti et al demonstrated that either zoledronic acid ( $4 \mathrm{mg}$ ) administered as a single intravenous regimen or neridronate $(200 \mathrm{mg})$ given intravenously at the dose of $100 \mathrm{mg}$ for 2 consecutive days showed a similar short-term efficacy in achieving biochemical remission at 6 and 9 months in up to $90 \%$ of patients with $\mathrm{PDB}$, who did not respond to pamidronate therapy. ${ }^{27}$ On the basis of the results obtained in this study, the authors consequently performed a randomized open-label study evaluating the long-term efficacy of the same neridronate dose $(200 \mathrm{mg})$, either administered as an intravenous or intramuscular regimen, in 56 patients with $\mathrm{PDB}$, with a 3-year follow-up. ${ }^{28}$ After 6 months, $92.6 \%$ of patients receiving intravenous neridronate and $96.5 \%$ of patients with intramuscular regimen achieved a therapeutic response in terms of normalization of alkaline phosphatase levels or reduction of at least $75 \%$ in total alkaline phosphatase excess (Figure 2). Response rates were maintained at 12 months but decreased progressively at 24 and 36 months in both neridronate regimens. At 6 months from treatment, $77.8 \%$ of patients in the intravenous regimen, and $86.2 \%$ of those in the intramuscular one, reported a decrease of bone pain at pagetic sites. 


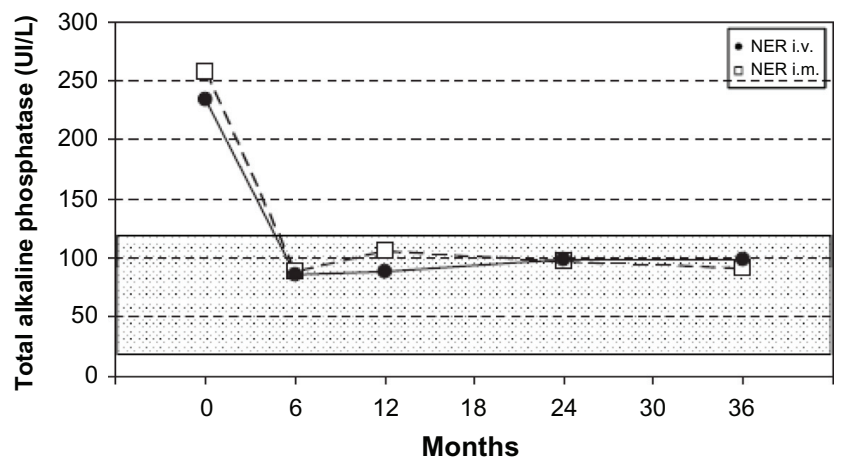

Figure 2 Mean serum alkaline phosphatase levels during the 36-month study for the intramuscular and intravenous neridronate regimens.

Notes: The shaded area represents the normal range of serum alkaline phosphatas. Copyright (c) 2011 American Society for Bone and Mineral Research. Merlotti D, Rendina D, Gennari L, et al. Comparison of Intravenous and Intramuscular neridronate regimens for the treatment of Paget disease of bone. J Bone Miner Res. 201।;26(3):512-518. ${ }^{28}$

Abbreviations: NER, neridronate; i.v., intravenous; i.m., intramuscular.

Overall, the long-term tolerability of both neridronate regimens was excellent and comparable with that of the previous short-term studies with intravenous infusion. The intramuscular regimen in particular was associated mainly with fever as the major symptom of the acute-phase reaction, while intravenous administration was associated more frequently with fever and muscular pain. Both routes of administration of the $200 \mathrm{mg}$ neridronate proved to have similar efficacy in the treatment of PDB. The intramuscular course of administration could be particularly useful for those patients unwilling or unable to undergo intravenous infusions or to take oral bisphosphonates, and represents an advantage as home treatment without major complications.

\section{Postmenopausal osteoporosis}

Bisphosphonates are the treatment of choice for postmenopausal as well as male and glucocorticoid (GC)-induced osteoporosis. Two pilot randomized-controlled studies evaluated the effect of neridronate on BMD. In the first study, performed in 78 women with postmenopausal osteoporosis, ${ }^{29}$ neridronate $(50 \mathrm{mg})$ was administered intravenously once every 2 months over 2 years and induced a significant and relevant increase in BMD $(+7.4 \%$ and $+5.8 \%$ at the lumbar spine and femoral neck, respectively). Similar results were obtained in the second pilot study, in which 40 postmenopausal women were treated with $25 \mathrm{mg}$ neridronate monthly intramuscularly. The densitometric changes were $6.6 \%$ and $4.2 \%$ at the spine and hip, respectively. ${ }^{30}$

A dose-finding clinical, multicenter trial ${ }^{31}$ in 188 postmenopausal osteoporotic women randomized to intramuscular treatment with $25 \mathrm{mg}$ neridronate every 2 weeks, neridronate 12.5 or $25 \mathrm{mg}$ every 4 weeks, or placebo showed a significant dose-response relationship over the three doses for the BMD changes at the total hip and for serum C-terminal telopeptide of type I collagen changes but not for spine BMD and bAP changes. These results indicate that the $25 \mathrm{mg}$ neridronate given intramuscularly monthly is probably the dose providing the maximum effects.

A recent ancillary study of this randomized controlled trial evaluated the changes in serum levels of the Wnt signaling antagonists sclerostin or dickkopf-1 (DKK1) during monthly intramuscular neridronate $(12.5,25.0$, or $50.0 \mathrm{mg})$ treatment. ${ }^{32}$ While serum DKK-1 remained stable during the entire 12-month study period in the three groups, serum sclerostin increased versus placebo group gradually and significantly only in patients treated with 25 or $50 \mathrm{mg}$ neridronate monthly. The changes in serum sclerostin at 12 months were negatively correlated $(P<0.001)$, with changes in bAP (a bone-formation marker) even when data were adjusted for serum C-terminal telopeptide of type I collagen (a boneresorption marker) changes and only when treated patients were included. This observation supports the hypothesis that the decrease in bone formation seen after several months of bisphosphonate therapy is associated with an increase in serum levels of sclerostin. ${ }^{32}$ These results suggest that Wnt signaling may play a role in the coupling between resorption and formation as it also emerges from studies on other osteometabolic drugs such as teriparatide ${ }^{33}$ and denosumab. ${ }^{34}$

\section{GC-induced osteoporosis}

Another type of osteoporosis is that induced by GCs. As a matter of fact, the main effect of GCs on bone is the inhibition of osteoblast function leading to a decrease in bone formation, together with the interaction with biological membranes. Several studies and reports show a decrease in BMD and an increased risk of fractures during GC use. Bone loss, which takes place from the first phase of GC treatment and mainly occurs in the first 6 months, is predominant in bone with a high trabecular content (eg, vertebrae). ${ }^{35} \mathrm{~A}$ long-standing use of GCs is typically indicated for rheumatic diseases such as rheumatoid arthritis, polymyalgia rheumatica, Sjögren's syndrome, systemic lupus erythematosus, and other types of arthritis.

Noteworthy is that almost $30 \%-50 \%$ of rheumatic patients on long-term GC treatment develop GC-induced osteoporosis (GIO) and osteoporosis-related fractures. ${ }^{35}$ In rheumatic patients, the diagnostic threshold in GIO, based on BMD measurements, is different from the established guidelines for postmenopausal osteoporosis. In fact, patients treated with GCs, even when showing BMD levels similar to those of nontreated subjects, have a higher risk of fractures. ${ }^{36}$ 
Prevention of GIO preferably relies on treatment with oral bisphosphonates or with intravenous zoledronate, but in patients with gastric or esophageal disease, or in those intolerant to oral bisphosphonates, especially when hospitalization is difficult, neridronate might represent a possible alternative. In fact, neridronate ( $25 \mathrm{mg}$ once monthly given intramuscularly) has also been tested in patients with GC-induced osteoporosis. Sixty-nine osteopenic and osteoporotic patients, affected by rheumatic diseases under chronic low-dose GC therapy and with gastric or esophageal conditions which contraindicated treatment with oral bisphosphonates, were randomly assigned to be treated with monthly intramuscular neridronate $25 \mathrm{mg}$ or placebo. (All patients also took daily calcium at the dose of $1000 \mathrm{mg}$ and vitamin D at the dose of $800 \mathrm{IU}$.) ${ }^{37}$ The trial showed that a 12-month intramuscular neridronate treatment in this kind of patient improves lumbar and femoral BMD $(+6.3 \%$ and $+4.2 \%$ versus placebo at the lumbar and femoral neck respectively; $P \leq 0.01$ ) and reduces the markers of bone resorption. ${ }^{37}$ These results appear comparable to those obtained in rheumatic patients under GC therapy with the administration of oral risedronate and alendronate.

Thanks to the proven safety, as observed for pediatric patients affected by OI, and the high gastrointestinal tolerability related to the parenteral administration, neridronate can be considered a first-choice treatment for GIO occurring in inflammatory bowel disease patients. Diamanti et al reported the case of a pediatric Crohn's disease patient who experienced GIO and related back pain 3 years after treatment with GCs. ${ }^{38}$ Intravenous neridronate was started after the first dual-energy X-ray absorptiometry evaluation, diluted in $250 \mathrm{~mL}$ of saline solution and infused intravenously over 30 minutes. A total of three infusions were administered, which were well tolerated without side effects. After the third administration of neridronate, a total spine radiograph indicated an improved bone density and a recovered vertebral height. So far, back pain has not reappeared..$^{38}$

\section{Complex regional pain syndrome type I (CRPS-I)}

A plethora of names has been used to describe this syndrome (eg, reflex sympathetic dystrophy, causalgia, Sudeck's atrophy, algodystrophy, neurodystrophy, post-traumatic dystrophy). Since the underlying pathophysiology is poorly understood, and no treatment has been found to be so effective to obtain the indication for this disease, CRPS-I still remains a matter of debate. CRPS-I is a severely disabling pain syndrome characterized by allodynia, hyperalgesia, edema, signs of vasomotor instability, movement disorders, joint stiffness, and regional osteopenia that in most cases develop following a trauma or surgery. Recently, Tran et al, by reviewing the literature published between 1950 and 2009, identified 41 randomized controlled trials which were suitable for their inclusion criteria. ${ }^{39}$ Eighteen of those studies included the use of pharmacological therapies. A single intravenous infusion of $7.5 \mathrm{mg}$ alendronate, ${ }^{40}$ intravenous clodronate $(300 \mathrm{mg}$ for 10 days), ${ }^{41}$ intravenous pamidronate (60 mg once), ${ }^{42}$ and $40 \mathrm{mg}$ daily oral alendronate for $12-16$ weeks $^{43}$ have been reported to be associated with positive results in controlling pain, edema, and functional impairment. ${ }^{44}$ However, none of these studies provided conclusive evidence of efficacy and sufficient data to make the use of a bisphosphonate formally indicated for the treatment of CRPS-I, mainly because of their limited size, with 10-20 treated patients per study.

A recent multicenter, randomized, double-blind placebocontrolled trial tested the efficacy of neridronate in patients with CRPS-I. ${ }^{45}$ The study involved 82 patients with CRPS-I at either hand or foot who were randomly assigned to intravenous infusion of $100 \mathrm{mg}$ neridronate given every third day four times, starting from day 1 (first infusion) and ending on day 10 (fourth infusion) or placebo. After 50 days, the placebo group was treated with the same regimen of neridronate (open-extension phase). Within the first 20 days, the visual analog scale (VAS) score decreased significantly from baseline more in the neridronate group. In the following 20 days, VAS remained unchanged in the placebo group and further decreased in the active group (Figure 3). A number of other indices of pain and quality of life were significantly improved with respect to the placebo group, and most of the patients in the active group were healed ${ }^{45}$ During the openextension phase in the placebo group, the results of treatment were comparable to those seen during the blind phase in the active group (Figure 4). A year later, none of the patients was

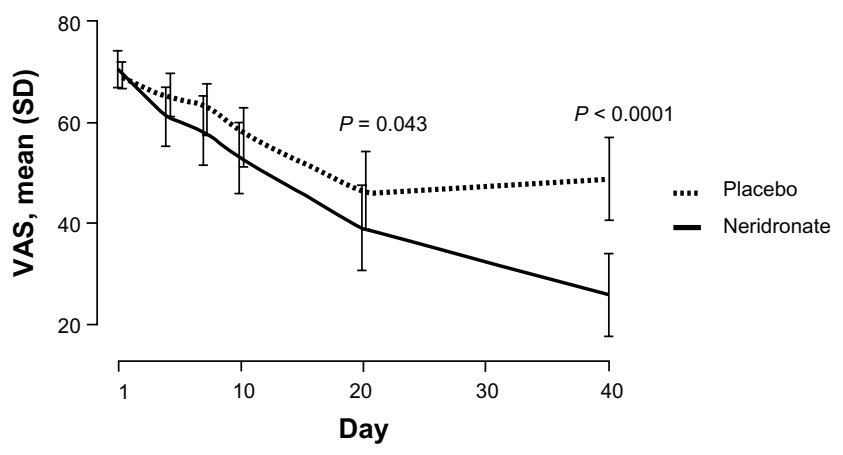

Figure 3 Double-blind phase: VAS trends from baseline to day 40 in patients with complex regional pain syndrome type I treated with neridronate or placebo.

Note: Varenna M, Adami S, Rossini M, et al. Treatment of complex regional pain syndrome type I with neridronate: a randomized, double-blind, placebo-controlled study. Rheumatology. 2013;52(3):534-542, by permission of Oxford University Press. ${ }^{45}$ Abbreviations: VAS, visual analog scale; SD, standard deviation. 


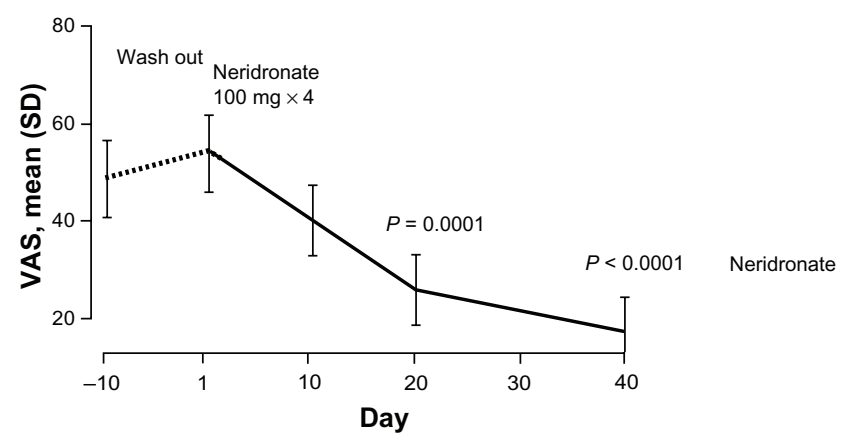

Figure 4 VAS values at the end of the follow-up period of the double-blind phase $($ day -10$)$ and after the treatment course with intravenous neridronate in patients with complex regional pain syndrome type $I$.

Note: Varenna M, Adami S, Rossini M, et al. Treatment of complex regional pain syndrome type I with neridronate: a randomized, double-blind, placebo-controlled study. Rheumatology. 201 3;52(3):534-542, by permission of Oxford University Press. ${ }^{45}$ Abbreviations: VAS, visual analog scale; SD, standard deviation.

referring symptoms linked to CRPS-I. These results provide conclusive evidence that the use of neridronate is associated with clinically relevant and persistent benefits and represents the treatment of choice for CRPS-I. ${ }^{45}$

\section{Other conditions}

\section{Thalassemia}

Osteopenia and osteoporosis are important causes of morbidity in patients with $\beta$-thalassemia major. In the pathogenesis of thalassemia-induced osteoporosis, genetic and acquired factors have been recognized. ${ }^{46}$ Notwithstanding optimal therapeutic regimens, effective iron chelation therapy and adequate hormone replacement, unbalanced bone turnover and active bone resorption remain a major issue. ${ }^{47-49}$ The increased boneturnover rate observed in thalassemic patients justifies the use of powerful antiresorptive drugs, such as bisphosphonates.

Treatment with intravenous neridronate was well tolerated and increased BMD in a large randomized trial involving 118 adults with $\beta$-thalassemia-induced osteoporosis. ${ }^{50}$ Patients were randomized to calcium (500 mg) and vitamin D (400 UI) daily and neridronate (100 mg intravenously every 3 months) or calcium and vitamin D alone. Significant increases in BMD at the lumbar spine and total hip were noted in the neridronate group at 6 and 12 months from baseline $(P<0.001)$, and values were significantly higher than the control group at both time intervals. As expected, neridronate also induced a significant reduction of bone-turnover markers. Improvement in quality of life assessed as reduction in back pain and analgesic use became evident after only 3 months of therapy. ${ }^{50}$

\section{Primary hyperparathyroidism (PHPT)}

The decrease in BMD and the moderate increase of fracture risk are often the only clinical manifestations of PHPT. ${ }^{51}$
In those patients not suitable, or unwilling, to undergo surgery, treatment with antiresorptive agents is a reasonable option. In a recent study, Rossini et al tested the effect of intravenous administration of neridronate (100 mg every 2 months for 2 years) on BMD and biochemical markers of bone metabolism in postmenopausal women with PHPT. ${ }^{51}$ Patients were followed up for 2 years after therapy discontinuation. Neridronate was well tolerated. BMD progressively rose to $6.6 \% \pm 7.6 \%, 2.9 \% \pm 4.5 \%$, and $5.0 \% \pm 3.9 \%$ at the lumbar spine, femoral neck, and total hip, respectively. The mean peak value was achieved 6 months after the last infusion at month 24. BMD values decreased during follow-up; however, 2 years after treatment discontinuation, lumbar spine BMD remained 3.9\% $\pm 5.5 \%$ higher than baseline values $(P<0.01)$, while femoral BMD returned to baseline. As regards to bone-turnover markers, bAP and serum C-telopeptide of type I collagen decreased significantly within 6 months, the nadir value being after 18-24 months $(P<0.01)$. After 6 months of therapy discontinuation, all these bone-turnover markers returned to baseline.

\section{Periodontitis}

Periodontitis is a multifactorial chronic infectious disease characterized by a loss of the connective tissue attachment to the teeth and the resorption of the alveolar bone due to the inflammatory processes. The etiology of periodontal disease is reducible to bacterial infection, which results in an inflammatory reaction. Tissue damage is generated by collagenolytic enzymes such as matrix metalloproteinases (MMPs) which significantly contribute to periodontal tissue damage. Therapies aimed at blocking tissue damage mediated by MMPs and at blocking alveolar bone destruction are actively being sought. With this scope, bisphosphonates, due to their action on bone metabolism, and in some cases to their downregulation of MMPs enzymes, are currently being investigated as adjunctive therapy in the management of periodontal disease.

In an open-label, randomized, clinical trial, Graziani et al investigated the effect of the addition of intramuscular administration of neridronate ( $12.5 \mathrm{mg}$ weekly for 3 months) in patients with advanced generalized chronic periodontal disease treated with conventional nonsurgical periodontal therapy as compared with the one obtained with conventional treatment alone. ${ }^{52}$ After discontinuation of treatment, patients were followed-up for 3 months. Although at the end of the study both groups showed improvements at sites with deep pocket depth, no difference was observed between patients treated with conventional nonsurgical therapy and conventional nonsurgical therapy with addition 
of neridronate. As argued by the authors, one of the possible reasons for the lack of clinical effects 3 months after the end of drug intake could be related to an insufficient dosage used to expect an effective action on the osseous metabolism of the alveolar bone.

\section{Malignancy}

Bisphosphonates had been used for years as standard therapy of bone diseases related to malignancy. In breast cancer patients with bone metastasis, several bisphosphonates demonstrated clinical efficacy. Zoledronic acid was the most extensively studied bisphosphonate in patients with bone metastases from prostate cancer or other solid tumors, and it remains the only bisphosphonate registered worldwide for these indications. ${ }^{53}$ In patients with multiple myeloma, bisphosphonate treatment reduces the risk of pathological vertebral fractures, skeletal-related morbidity, and pain. ${ }^{54}$ The bisphosphonates most often used are ibandronate, pamidronate, and zoledronate. The data on neridronate are rather limited. In seven patients with bone lesions for multiple myeloma, $100 \mathrm{mg}$ intravenous neridronic acid, monthly over a year, was associated with significant increases in spine BMD and decreases in bone-turnover markers. ${ }^{55}$ In patients with hypercalcemia owing to solid metastatic tumors, an infusion of $125 \mathrm{mg}$ neridronic acid (dissolved in $500 \mathrm{~mL}$ of saline solution) significantly decreased serum calcium inducing to normalization in $65 \%$ of the patients. ${ }^{56}$

Currently, the data are insufficient to extend the use of neridronate for these indications, although in-vitro studies ${ }^{57,58}$ and preliminary data on pediatric populations ${ }^{59}$ could open new opportunities in the future.

\section{Conclusion}

Neridronate is an aminobisphosphonate licensed for the treatment of $\mathrm{OI}$ and PDB. Its chemical structure and potency is very close to that of pamidronic acid. The results of a recent randomized, well powered clinical trial for the treatment of CRPS-I were very encouraging and should soon lead to a license for a new indication. Neridronate may be administered both intravenously and intramuscularly. This latter regimen is of particular interest and so far unique, allowing homecare treatments. According to the accumulated experiences from studies carried out in children with OI, this is to date the only bisphosphonate that can be used in children and in newborns.

\section{Disclosure}

Davide Gatti and Maurizio Rossini have received speaking fees from Amgen, Abiogen, Merck, Eli-Lylli, and Pfizer.
Ombretta Viapiana has received speaking fees from Abiogen, Merck, and Pfizer. Luca Idolazzi has received speaking fees from Novartis. Silvano Adami is consultancy honoraria for Merck, and Amgen, and has received speaking fees from Amgen, Abiogen, Merck, Eli-Lylli, Pfizer, Novartis, and GSK.

\section{References}

1. Gatti D, Viapiana O, Idolazzi L, Fracassi E, Adami S. Neridronic acid for the treatment of bone metabolic diseases. Expert Opin Drug Metab Toxicol. 2009;5(10):1305-1311.

2. Ebetino FH, Hogan AM, Sun S, et al. The relationship between the chemistry and biological activity of the bisphosphonates. Bone. 2011;49(1):20-33.

3. Ebetino FH, Bayless AV, Amburgey J, Ibbotson KJ, Dansereau S, Ebrahimpour A. Elucidation of a pharmacore for the bisphosphonate mechanism of bone antiresorptive activity. Phosphorus Sulfur Silicon Relat Elem. 1996;109(1-4):217-220.

4. Amin D, Cornell SA, Gustafson SK, et al. Bisphosphonates used for the treatment of bone disorders inhibit squalene synthase and cholesterol biosynthesis. J Lipid Res. 1992;33:1657-1663.

5. Graham DY, Malaty HM, Goodgame R. Primary amino-bisphosphonates: a new class of gastrotoxic drugs-comparison of alendronate and aspirin. Am J Gastroenterol. 1997;92(8):1322-1325.

6. Lin JH. Bisphosphonates: a review of their pharmacokinetic properties. Bone. 1996;18(2):75-85.

7. Devogelaer JP, Malghem J, Maldague B, Nagant de Deuxchaisnes C. Radiological manifestations of bisphosphonate treatment with APD in a children suffering from osteogenesis imperfecta. Skeletal Radiol. 1987;16(5):360-363.

8. Rauch F, Glorieux FH. Osteogenesis imperfecta. Lancet. 2004; 363(9418):1377-1385.

9. Glorieux FH, Bishop NJ, Plotkin H, Chabot G, Lanoue G, Travers R. Cyclic administration of pamidronate in children with severe osteogenesis imperfecta. N Engl J Med. 1998;339(14):947-952.

10. Land C, Rauch F, Munns CF, Sahebjam S, Glorieux FH. Vertebral morphometry in children and adolescents with osteogenesis imperfecta: effect of intravenous pamidronate treatment. Bone. 2006;39(4): 901-906.

11. Plotkin H, Rauch F, Bishop NJ, et al. Pamidronate treatment of severe osteogenesis imperfecta in children under 3 years of age. J Clin Endocrinol Metab. 2000;85(5):1846-1850.

12. Gatti D, Antoniazzi F, Prizzi R, et al. Intravenous neridronate in children with osteogenesis imperfecta: a randomized controlled study. J Bone Miner Res. 2005;20(5):758-763.

13. Antoniazzi F, Zamboni G, Lauriola S, Donadi L, Adami S, Tatò L. Early bisphosphonate treatment in infants with severe osteogenesis imperfecta. J Pediatr. 2006;149(2):174-179.

14. Adami S, Gatti D, Colapietro F, et al. Intravenous neridronate in adults with osteogenesis imperfecta. J Bone Miner Res. 2003;18(1):126-230.

15. Semler O, Beccard R, Palmisano D, et al. Reshaping of vertebrae during treatment with neridronate or pamidronate in children with osteogenesis imperfecta. Horm Res Paediatr. 2011;76(5):321-327.

16. Marini JC, Bordenick S, Heavner G, et al. The growth hormone and somatomedin axis in short children with osteogenesis imperfecta. J Clin Endocrinol Metab. 1993;76(1):251-256.

17. Antoniazzi F, Bertoldo F, Mottes M, et al. Growth hormone treatment in osteogenesis imperfecta with quantitative defect of type I collagen synthesis. J Pediatr. 1996;129(3):432-439.

18. Marini JC, Hopkins E, Glorieux FH, et al. Positive linear growth and bone responses to growth hormone treatment in children with types III and IV osteogenesis imperfecta: high predictive value of the carboxyterminal propeptide of type I procollagen. J Bone Miner Res. 2003;18(2): $237-243$. 
19. Antoniazzi F, Monti E, Venturi G, et al. GH in combination with bisphosphonate treatment in osteogenesis imperfecta. Eur J Endocrinol. 2010;163(3):479-487.

20. Gutta R, Louis PJ. Bisphosphonates and osteonecrosis of the jaws: science and rationale. Oral Surg Oral Med Oral Pathol Oral Radiol Endod. 2007;104(2):186-193.

21. Maines E, Monti E, Doro F, Morandi G, Cavarzere P, Antoniazzi F. Children and adolescents treated with neridronate for osteogenesis imperfecta show no evidence of any osteonecrosis of the jaw. $J$ Bone Miner Metab. 2012;30(4):434-438.

22. Falchetti A, Marini F, Masi L, Amedei A, Brandi ML. Genetic aspects of the Paget's disease of bone: concerns on the introduction of DNAbased tests in the clinical practice. Advantages and disadvantages of its application. Eur J Clin Invest. 2010;40(7):655-667.

23. Adami S, Bevilacqua M, Broggini M, et al. Short-term intravenous therapy with neridronate in Paget's disease. Clin Exp Rheum. 2002; 20(1):55-58.

24. Walsh JP, Ward LC, Stewart GO, et al. A randomized clinical trial comparing oral alendronate and intravenous pamidronate for the treatment of Paget's disease of bone. Bone. 2004;34(4):747-754.

25. Reid IR, Miller P, Lyles K, et al. Comparison of a single infusion of zoledronic acid with risedronate for Paget's disease. $N$ Engl J Med. 2005;353(9):898-908

26. Hosking D, Lyles K, Brown JP, et al. Long-term control of bone turnover in Paget's disease with zoledronic acid and risedronate. J Bone Miner Res. 2007;22(1):142-148.

27. Merlotti D, Rendina D, Gennari L, et al. Comparison of different intravenous bisphosphonate regimens for Paget's disease of bone. J Bone Miner Res. 2007;22(10):1510-1517.

28. Merlotti D, Rendina D, Gennari L, et al. Comparison of Intravenous and Intramuscular neridronate regimens for the treatment of Paget disease of bone. J Bone Miner Res. 2011;26(3):512-518.

29. Braga V, Gatti D, Colapietro F, et al. Intravenous intermittent neridronate in the treatment of postmenopausal osteoporosis. Bone. 2003;33(3):342-345.

30. Cascella T, Musella T, Orio F, et al. Effects of neridronate treatment in elderly women with osteoporosis. J Endocrinol Invest. 2005;28(3): 202-208.

31. Adami S, Gatti D, Bertoldo F, et al. Intramuscular neridronate in postmenopausal women with low bone mineral density. Calcif Tissue Int. 2008;83(5):301-307.

32. Gatti D, Viapiana O, Adami S, Idolazzi L, Fracassi E, Rossini M. Bisphosphonate treatment of postmenopausal osteoporosis is associated with a dose dependent increase in serum sclerostin. Bone. 2012;50(3): 739-742.

33. Gatti D, Viapiana O, Idolazzi L, Fracassi E, Rossini M. The waning of teriparatide effect on bone formation markers in postmenopausal osteoporosis is associated with increasing serum levels of DKK1. J Clin Endocrinol Metab. 2011;96(5):1555-1559.

34. Gatti D, Viapiana O, Fracassi E, et al. Sclerostin and DKK1 in postmenopausal osteoporosis treated with denosumab. J Bone Miner Res. 2012;27(11):2259-2263.

35. De Nijs RN. Glucocorticoid-induced osteoporosis: a review on pathophysiology and treatment options. Minerva Med. 2008;99(1): 23-43.

36. Canalis E, Bilezikian JP, Angeli A, Giustina A. Perspectives on glucocorticoid induced osteoporosis. Bone. 2006;34(4):593-598.

37. Benucci M, Saviola G, Baiardi P, et al. Effects of monthly intramuscular neridronate in rheumatic patients in chronic treatment with low-dose glucocorticoids. Clin Exp Rheumatol. 2009;27(4):567-573.

38. Diamanti A, Noto C, Basso MS, Papadatou B, Bracci F, Castro M. Efficacy and safety of intravenous neridronate in pediatric bone loss associated to Crohn's disease: a case report. Clin Exp Rheumatol. 2009;27(1):165.

39. Tran de QH, Duong S, Bertini P, Finlayson RJ. Treatment of complex regional pain syndrome: a review of the evidence. Can J Anaesth. 2010;57(2):149-166.
40. Adami S, Fossaluzza V, Gatti D, et al. Bisphosphonate therapy of reflex sympathetic dystrophy syndrome. Ann Rheum Dis. 1997;56: 201-204.

41. Varenna M, Zucchi F, Ghiringhelli D, et al. Intravenous clodronate in the treatment of reflex sympathetic dystrophy syndrome. A randomized, double blind, placebo controlled study. J Rheumatol. 2000;27: 1477-1483.

42. Robinson JN, Sandom J, Chapman PT. Efficacy of pamidronatein complex regional pain syndrome type I. Pain Med. 2004;5:276-280.

43. Manicourt DH, Brasseur JP, Boutsen Y, et al. Role of alendronate in therapy for post-traumatic complex regional pain syndrome type I of the lower extremity. Arthritis Rheum. 2004;50:3690-3697.

44. Brunner F, Schmid A, Kissling R, et al. Biphosphonates for the therapy of complex regional pain syndrome I - systematic review. Eur J Pain. 2009;13:17-21.

45. Varenna M, Adami S, Rossini M, et al. Treatment of complex regional pain syndrome type I with neridronate: a randomized, double-blind, placebo-controlled study. Rheumatology. 2013;52(3):534-542.

46. Haidar R, Musallam KM, Taher AT. Bone disease and skeletal complications in patients with $\beta$ thalassemia major. Bone. 2011;48(3): 425-432.

47. Lasco A, Morabito N, Gaudio A, Buemi M, Wasniewska M, Frisina N. Effects of hormonal replacement therapy on bone metabolism in young adults with beta-thalassemia major. Osteoporos Int. 2001;12(7): 570-575.

48. Karimi M, Ghiam AF, Hashemi A, Alinejad S, Soweid M, Kashef S. Bone mineral density in beta-thalassemia major and intermedia. Indian Pediatr. 2007;44(1):29-32.

49. Baldini M, Forti S, Marcon A, et al. Endocrine and bone disease in appropriately treated adult patients with beta-thalassemia major. Ann Hematol. 2010;89(12):1207-1213.

50. Forni GL, Perrotta S, Giusti A, et al. Neridronate improves bone mineral density and reduces back pain in $\beta$-thalassaemia patients with osteoporosis: results from a phase 2 , randomized, parallel-arm, open-label study. Br J Haematol. 2012;158(2):274-282.

51. Rossini M, Viapiana O, Kalpakcioglu B, et al. Long-term effects of neridronate and its discontinuation in patients with primary hyperparathyroidism. Calcif Tissue Int. 2011;89(1):21-28.

52. Graziani F, Cei S, Guerrero A, et al. Lack of short-term adjunctive effect of systemic neridronate in non-surgical periodontal therapy of advanced generalized chronic periodontitis: an open label-randomized clinical trial. J Clin Periodontol. 2009;36(5): 419-427.

53. Mackiewicz-Wysocka M, Pankowska M, Wysocki PJ. Progress in the treatment of bone metastases in cancer patients. Expert Opin Investig Drugs. 2012;21(6):785-795.

54. Mhaskar R, Redzepovic J, Wheatley K, et al. Bisphosphonates in multiple myeloma: a network meta-analysis. Cochrane Database Syst Rev. 2012;5:CD003188.

55. Pittari G, Costi D, Raballo M, et al. Intravenous neridronate for skeletal damage treatment in patients with multiple myeloma. Acta Biomed. 2006;77:81-84.

56. O’Rourke NP, McCloskey EV, Rosini S, et al. Treatment of malignant hypercalcaemia with aminohexane bisphosphonate (neridronate). $\mathrm{Br} J$ Cancer. 1994;69:914-917.

57. Chebbi I, Migianu-Griffoni E, Sainte-Catherine O, Lecouvey M, Seksek O. In vitro assessment of liposomal neridronate on MDA-MB231 human breast cancer cells. Int J Pharm. 2010;383(1-2):116-122.

58. Nicolin V, Narducci P, Bareggi R. Role of neridronate on MCF-7 estrogen dependent breast cancer model of bone metastasis: a preliminary study. Invest New Drugs. 2011;29(1):189-191.

59. Clerico A, Paiano M, Suraci S, Marucci G, Zambrano A, Varrasso G. Intravenous neridronate in children affected by malignancies: preliminary results; Pediatric Blood \& Cancer; 39th Annual Conference of the International Society of Paediatric Oncology SIOP 2007, Mumbai, India, 31 October-3 November 2007, SIOP Abstracts; Volume 49, Issue Supplement 6, pages 513, 1 October 2007. 
Therapeutics and Clinical Risk Management

Dovepress

\section{Publish your work in this journal}

Therapeutics and Clinical Risk Management is an international, peerreviewed journal of clinical therapeutics and risk management, focusing on concise rapid reporting of clinical studies in all therapeutic areas, outcomes, safety, and programs for the effective, safe, and sustained use of medicines. This journal is indexed on PubMed Central, CAS,
EMBase, Scopus and the Elsevier Bibliographic databases. The manuscript management system is completely online and includes a very quick and fair peer-review system, which is all easy to use. Visit http://www.dovepress.com/testimonials.php to read real quotes from published authors.

Submit your manuscript here: http://www.dovepress.com/therapeutics-and-clinical-risk-management-journal 